\title{
A RESEARCH DEVICE TO CONTROL IGNITION AND INJECTION PARAMETERS FOR A SPARK-IGNITION ENGINE
}

\author{
Katarzyna Grochowska \\ Technical University of Lodz, Faculty of Mechanical Engineering, \\ Żeromskiego Street 116, 90-924 Lodz, Poland \\ tel.: +48513793754 \\ e-mail:kggerta@wp.pl
}

\begin{abstract}
This paper describes the possibility of controlling injection and ignition parameters of a spark-ignition engine performed with the author's controller. The modification of output signals according to given parameters of the interface is shown in this article. Simulations, supported by oscilloscope graphs, were performed using the controller designed to work with four-cylinder spark-ignition engine.

This device allows modifying the ignition and injection parameters depending on the values entered in the author's interface on the PC. This interface enables a serial transmission through a RS 232 serial port and sending data to the microcontroller. The controller allows for a measurement of the values of the sensors and a generation of output signals for the actuators.

The simulation done in this work involves the modification of injection and ignition angle parameters: ignition angle of advance (KWZ), injection angle of advance $(K W W)$ and time parameters: time of injection $(K W)$, time of ignition (KZ).

Also shown in this article are possibilities of adapting the controller of the spark-ignition engine to a simultaneous, batched and sequential injection mode.
\end{abstract}

Keywords: transport, road transport, combustion engines, spark-ignition engine, controlling

\section{Controller's principle}

The controller is based on the Atmega32 microcontroller timed with an internal $8 \mathrm{MHz}$ frequency timer. The author's program for this controller enables $\mu \mathrm{C}-\mathrm{PC}$ communication. It also can measure values from the engine sensors and generate output signals for the actuators. A ZL3AVR development kit was used for this project. The kit is equipped with an LCD display, connection to a ZL2PRG programmer and DB9 socket for serial transmission. Due to the incompatibility of the signals from the sensors and to the elements with the values of the current and voltage generated and adopted by the Atmega32 microcontroller, it is necessary to equip the controller with a hardware interface that will change the ranges of these values.

The Atmega32 microcontroller is equipped with: 4 ports: PA, PB, PC and PD -8 pins each, pin VCC (digital supply voltage), GND (for grounding), XTAL1 (input to the inverting oscillator amplifier and input to the internal clock operating circuit), XTAL2 (output from the inverting oscillator amplifier), AREF, AGND (analogue reference pins for the A/D Converter), AVCC (supply voltage pin for Port A and the A/D Converter), and a RESET button.

The crankshaft rotational speed sensor is connected to the PB.1 pin. Either an inductive (reluctance) sensor or Hall crankshaft position sensor can also be used. In the event of using an inductive sensor, it is necessary to apply a hardware-interface filtering signal. A sinusoidal-similar signal of variable amplitude and variable frequency, depending on the rotational speed, is converted to a rectangular wave signal with a variable frequency and amplitude of $5 \mathrm{~V}$. The measurement of the duration of one period enables to calculate the instantaneous rotational speed. The Hall sensor is factory-equipped with a converter, which causes the output signal to be rectangular. 


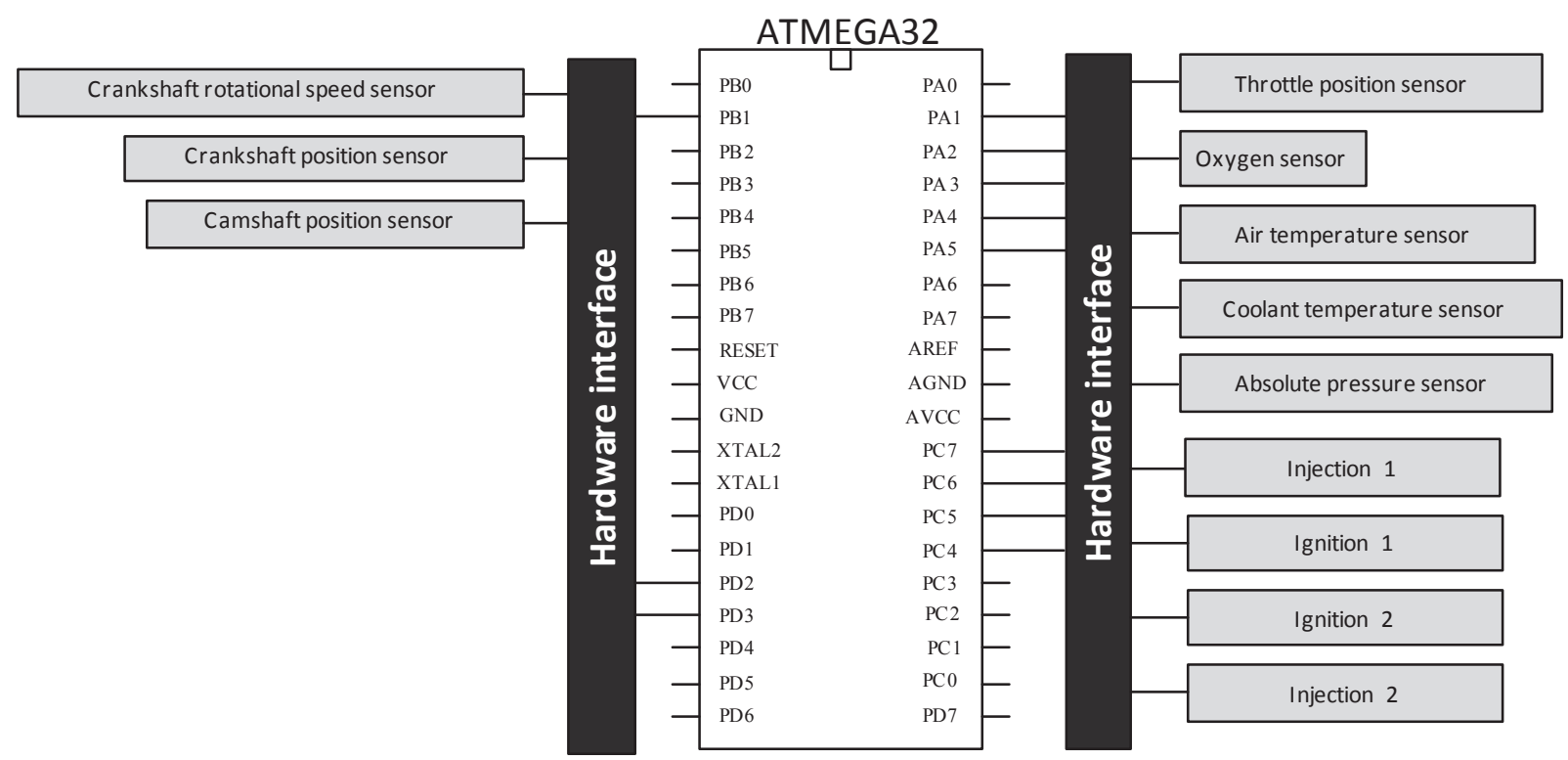

Fig. 1. Diagram showing how the actuators and sensors connect to processor Atmega32 for the batched injection mode

The crankshaft rotational speed is calculated similarly by determining the duration of one period, which is counted by the microcontroller's timer (counter).

The crankshaft position sensor is connected to the PD.2 pin(INT0) and generates a signal in a specific angular distance before the 1st cylinder reaches the TDC. This value is currently set to 108 degrees and can be easily changed in the program, whereby making it possible to modify the position of the marker.

The camshaft position sensor is connected to PD.3 INT1 pin.

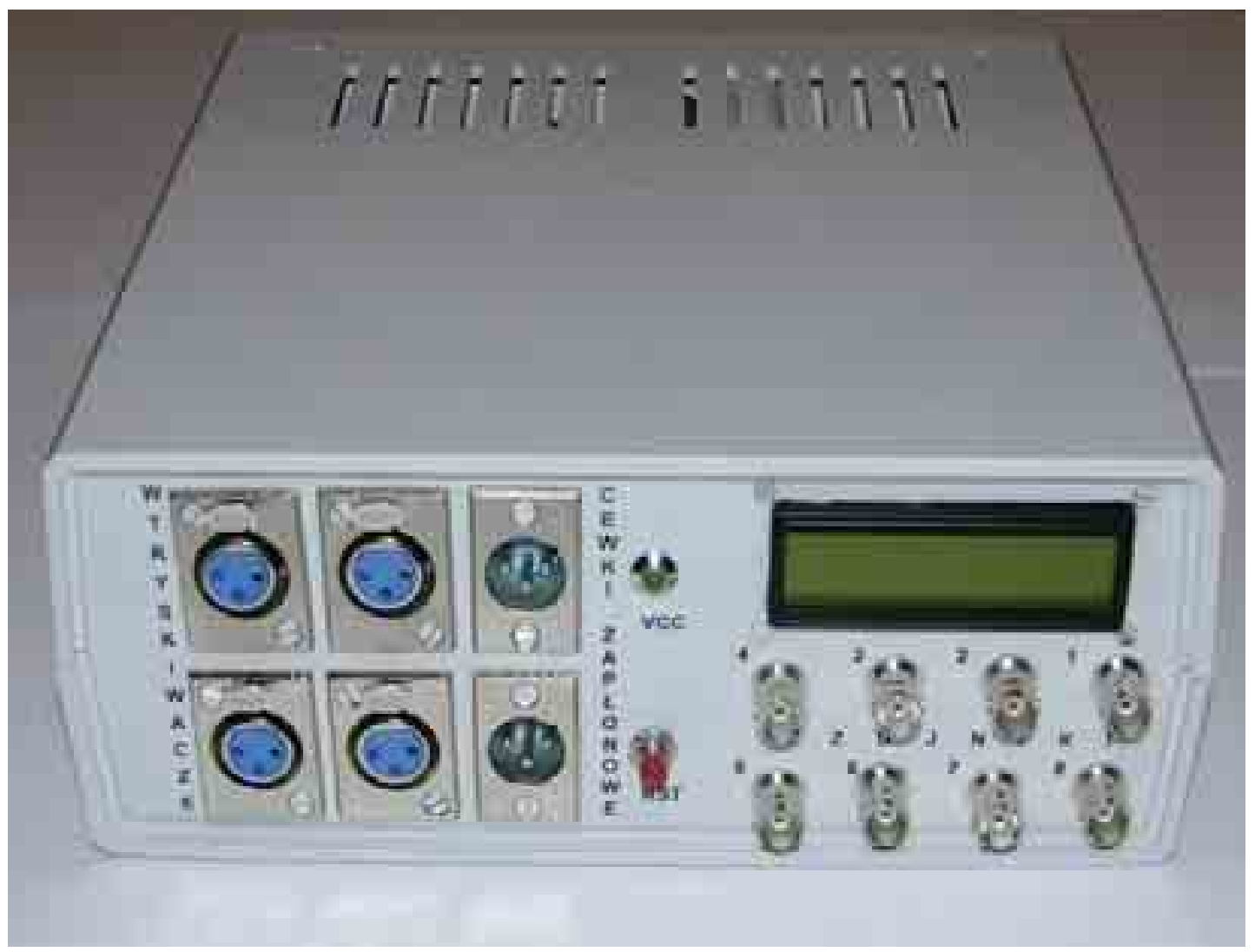

Fig. 2. Controller's front panel for batched injection 
Port PA is equipped with an analogue-digital converter allowing for a direct measurement of the values of the voltage from the throttle position sensor, air temperature sensor, coolant temperature sensor and absolute pressure sensor. These sensors are connected respectively to pins: PA.5, PA.3, PA.2 and PA.1.

The actuators comprises of the following: 2 dual-pole ignition coils (1st ignition coil for the 2nd and 3rd cylinder and the second for the 1st and 4th cylinder) and four injectors (connected to pins: PC.7, PC.6, PC.5 and PC.4) divided in two groups (two injectors each). The first group is assigned to the 3 rd and 4th cylinder and the second group to the 1st and 2nd cylinder.

With the help of interruptions it is possible to generate signals the ignition coils and injectors at a given time.

During engine operation, signals from the crankshaft rotational speed sensor and crankshaft position sensor are generated for a particular rotational speed. They become the main signals in manual mode and allow for calculation of the rotational speed, and thus enable the conversion of the angular parameters of the fuel injection and ignition (injection angle of advance KWW, ignition angle of advance KWZ) given by users from PC to time values. The generated signals are sent to the two dual-pole ignition coils and to the four injectors.

Modification of the program and hardware interface makes it possible to switch the injection mode to a simultaneous injection mode (Fig. 3), batched (Fig. 4) or sequential (Fig. 5).

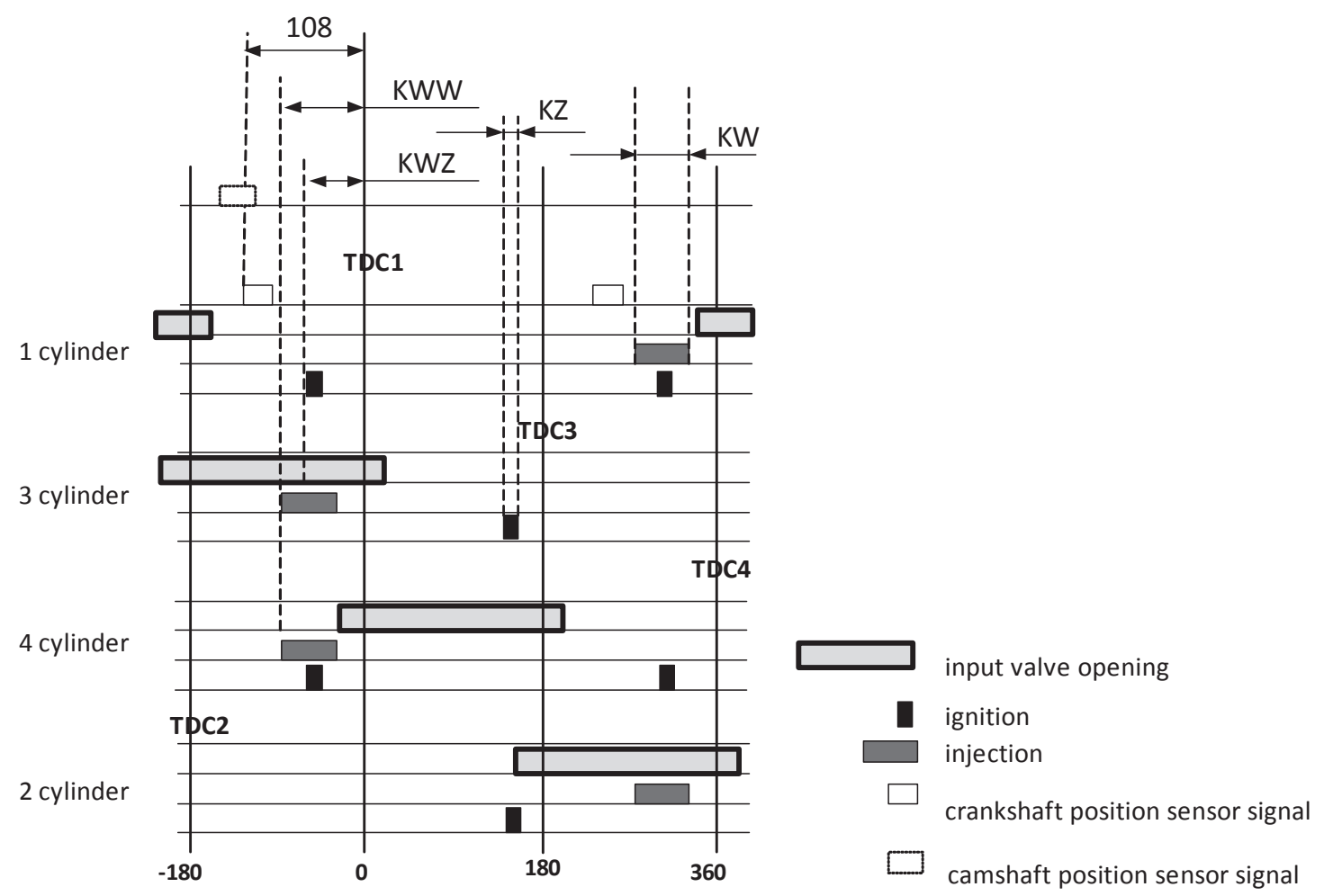

Fig. 3. Diagram of signals control for actuators in sequential mode

The written program in microprocessor's memory is divided into four parts. The first one applies to the serial communication with PC (sending and receiving of characters), while the second one allows the values of the sensors (including rotational speed and crankshaft position) which are connected directly to the controller, to be measured. The third is a calculation part that generates the parameters of the interrupts, and the fourth are interrupts.

In the beginning of the program, the initial values of all the parameters, which can be modified through the interface, are included. It allows the engine to start its operation without the risk of disturbances occurring before the values given by the user are given. 


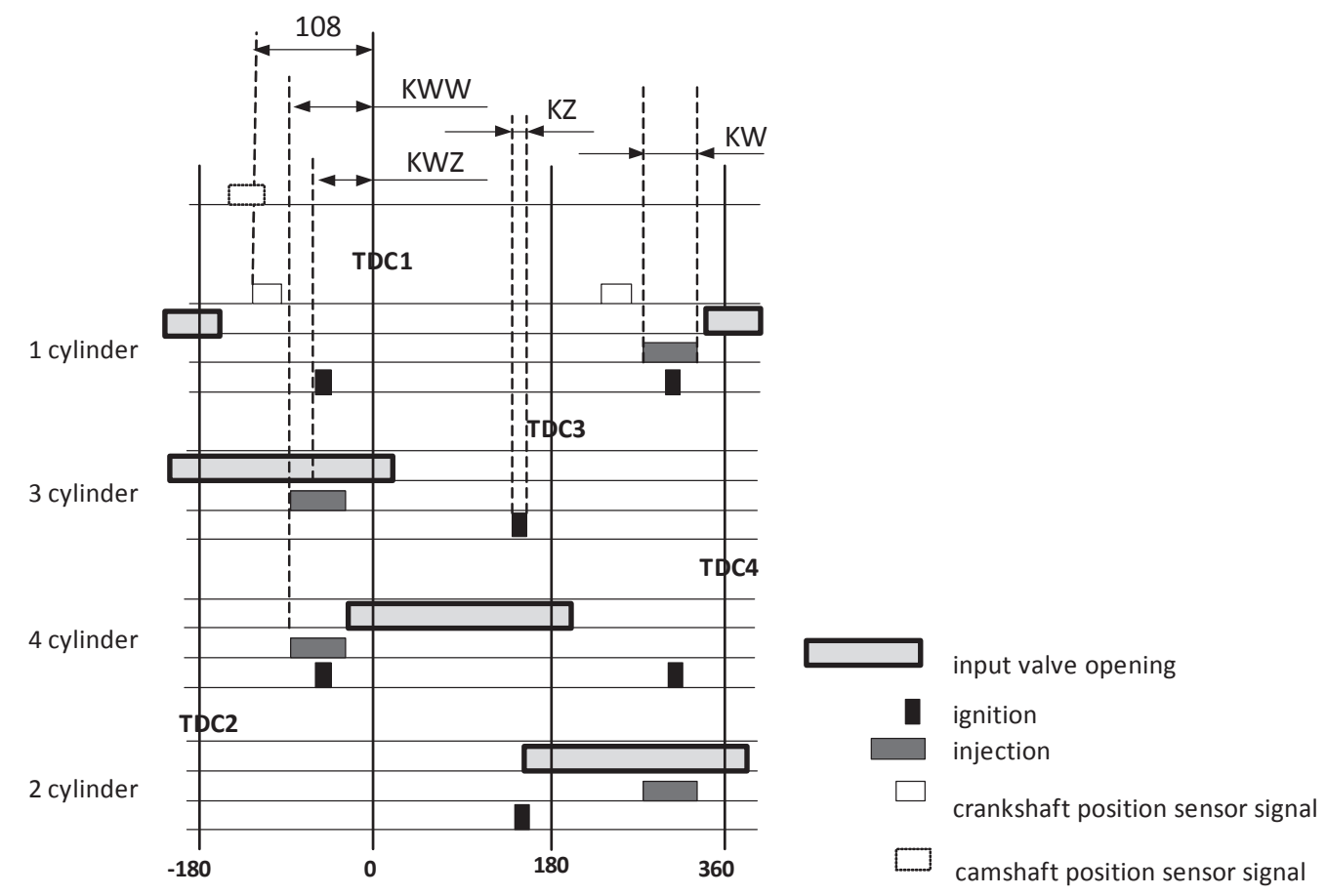

Fig. 4. Diagram of signals control for actuators in batched mode
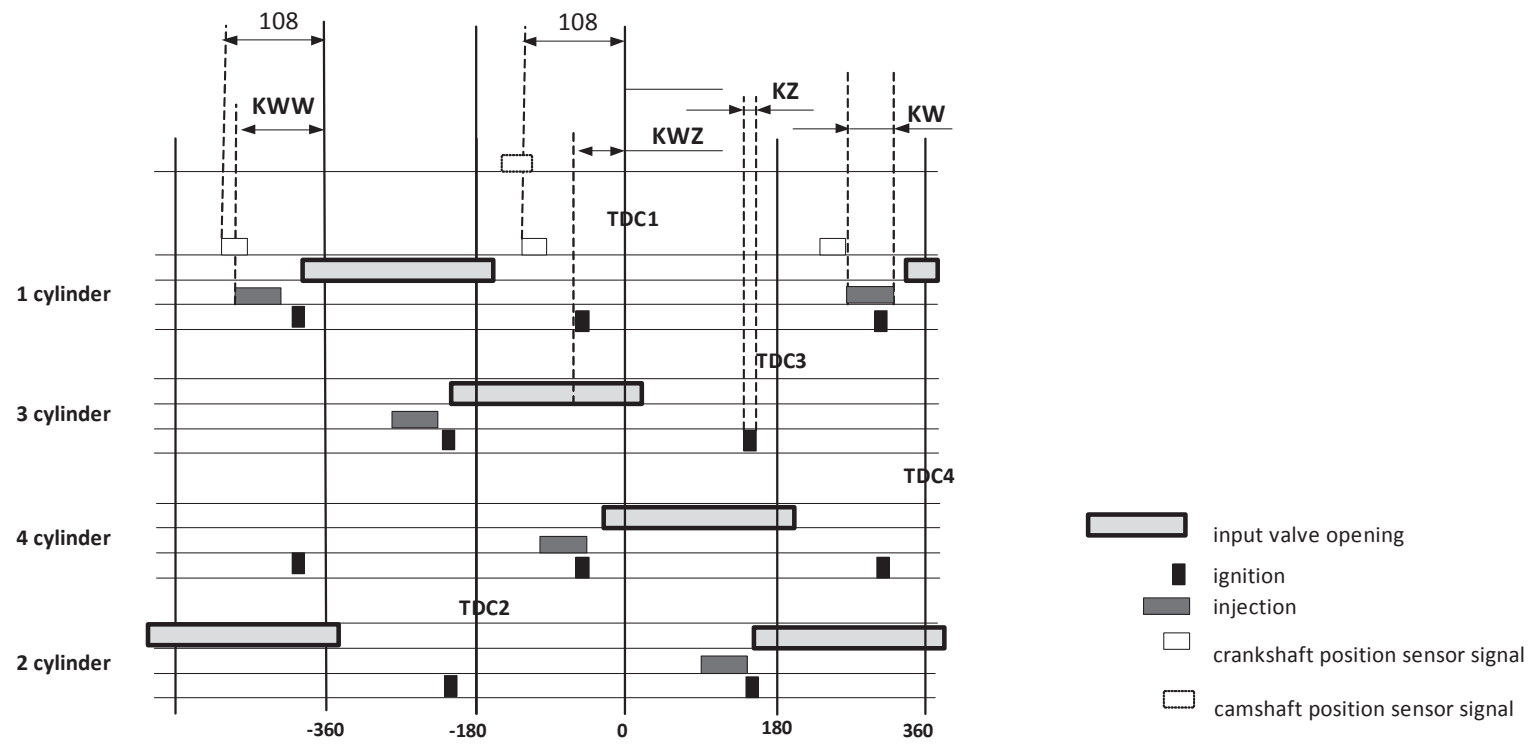

Fig. 5. Diagram of signals control for actuators in sequential mode

\subsection{Control of signals for batched injection}

During the simulation, the signals of the rotational speed sensor and crankshaft position sensor were produced from rectangular wave generator. This way we can obtain a signal similar to the signals generated by Hall rotational speed and position sensors.

By modifying the frequency, we can obtain signals equivalent to real signals generated by sensors for a particular rotational speed. The simulation provided an opportunity to operate in laboratory conditions for given rotational speed values.

Waveforms that are later recorded on oscilloscope graphs were obtained by entering the values of the ignition angle of advance KWZ, injection angle of advance $\mathrm{KWW}$, time of injection $\mathrm{KW}$ and angle of ignition KZ. 
Tab. 1. Comparison of oscilloscope graphs for the time parameters of ignition and injection: $K W=2000 \mu \mathrm{s}, 5000 \mu \mathrm{s}$, $9000 \mu \mathrm{s}$ and $\mathrm{KZ}=1500 \mu \mathrm{s}, 2500 \mu \mathrm{s}, 3500 \mu \mathrm{s}$

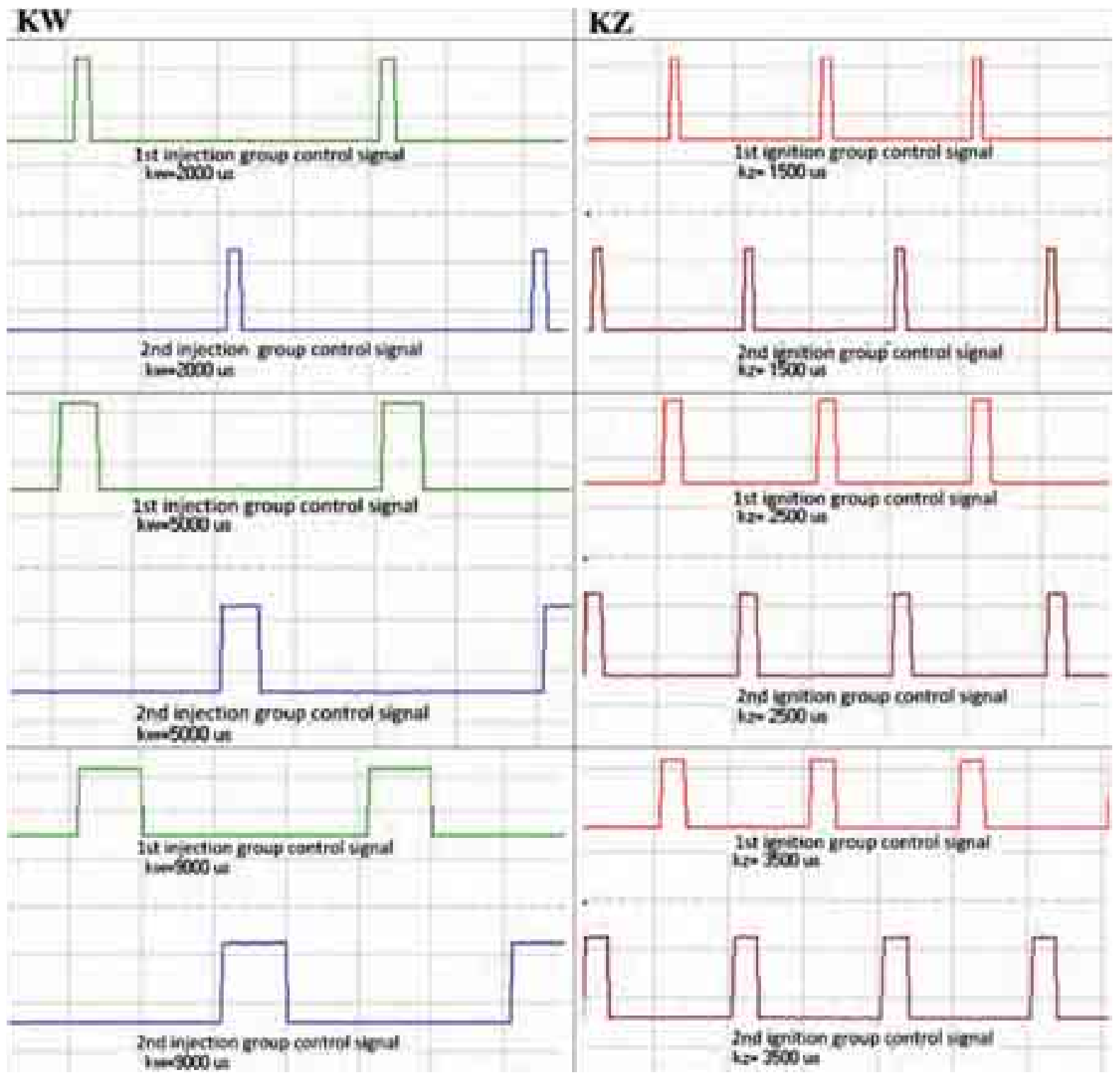

During simulation, the following values were given for KW: $2000 \mu \mathrm{s}, 5000 \mu$ s and $9000 \mu \mathrm{s}$. For the first group, the KW values were registered in a configuration in relation with the signal from the simulated crankshaft position sensor. The second group control signals were verified related to the simulated signal from the crankshaft position sensor by simultaneously registering the signal from the first and second group.

The graphs are presented in Tab. 1 in the first column marked as KW. Similar procedure was used for the KZ parameter for the following values: $1500 \mu \mathrm{s}, 2500 \mu \mathrm{s}$ and $3500 \mu \mathrm{s}$. In Tab. 1, the oscilloscope graphs are presented in the second column marked as KZ. Presented in this table is the comparison and confirmation of the controller's proper operation, in terms of controlling the length of the signals that control the injection and ignition actuators.

It has to be mentioned that the injection as well as ignition signals are generated independently. This way it is possible to move the signals in relation to one another on the time axis or even put the signals on each other at the same time. This can be achieved because the control signals are generated on different pins of port $\mathrm{C}$.

The second part of the simulation is the modification of the angle parameters: the injection angle of advance (KWW) and the ignition angle of advance (KWZ). 
Tab. 2. Comparison of oscilloscope graphs for injection and ignition angle parameters: $K W W=20,50,80 \mathrm{DCR}$ and $K W Z=20,30,40 \mathrm{DCR}$.

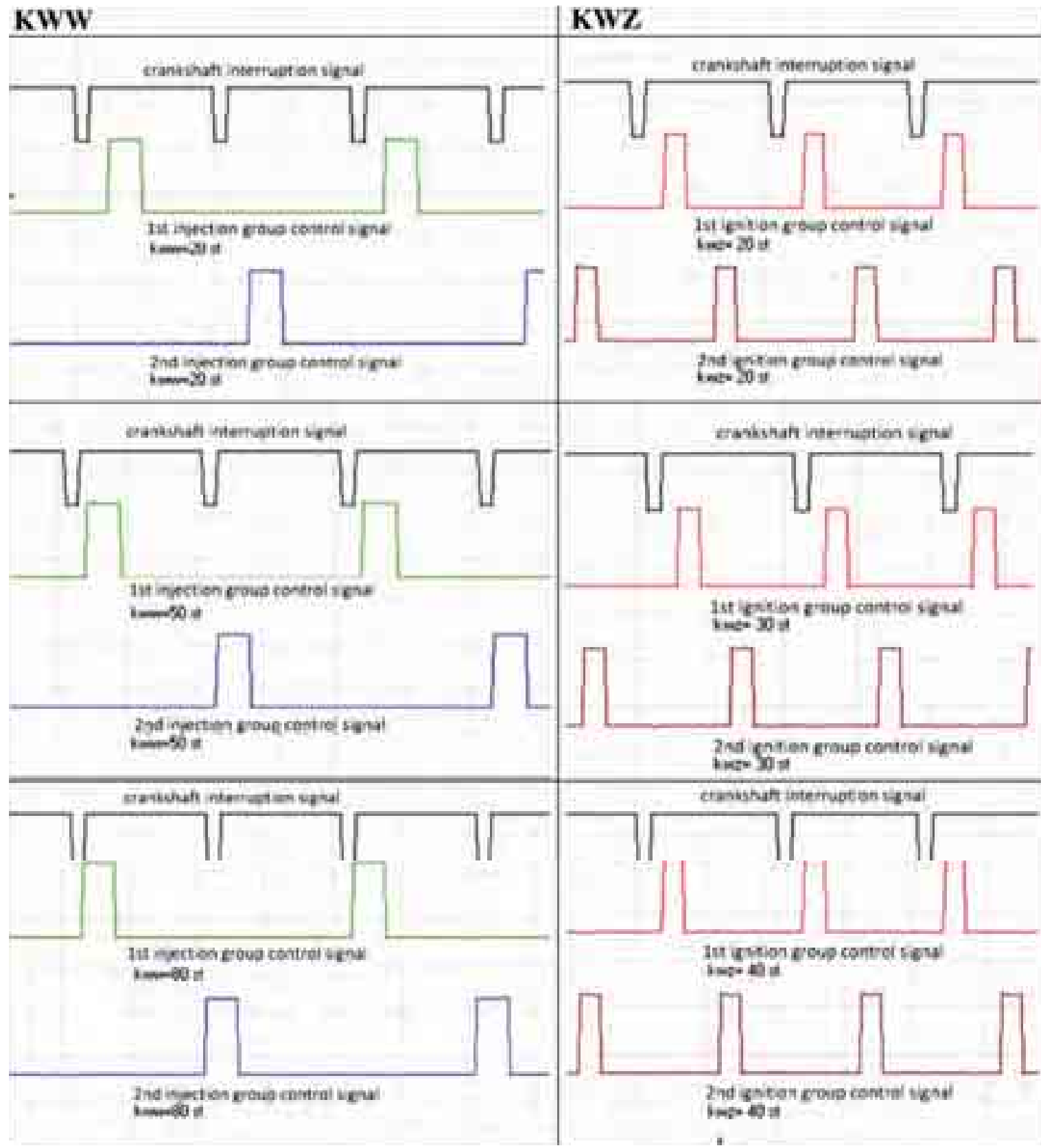

For the KWW parameter, the signals controlling the injectors of the first group were recorded in relation to the signal coming from the generator simulating the interrupt signal of the crankshaft position sensor. Then the second group of injectors in relation to the interrupt signal of the crankshaft position was recorded. For the publication purposes, the control signals of the first and the second groups were combined on the same oscilloscope graph. The signal was recorded for the following KWW values: 20, 50 and 80 DCR. These graphs have been placed in Tab. 2 in the first column marked as KWW.

Similarly, the oscilloscope graphs were made for the ignition angle of advance KWZ. The KWZ values set were as follows: 20,30 and 40 DCR. The results of this simulation are presented in Tab. 2 in the second column labelled KWZ.

In the case of the increasing value of the KWZ parameter, we can observe a shift of the signal controlling the first and second ignition coil in relation to the signal releasing the crankshaft interrupt. In addition, it can be seen that a constant distance between the signals controlling the first and second ignition coil can be maintained. 
With increasing values of the KWW parameter, we can also observe a shift to the left of the signals controlling the first and second injectors' group in relation to the interrupt signal, meanwhile maintaining the same distance between the beginning of injection of the first and second group.

For the KW and KZ parameters, it can be seen that with increasing values of the given parameter we receive a control signal of greater length, of which its time shown on the oscilloscope graph is equal to the value entered in the user interface of the PC.

Keeping equal distances between the signals of the first and the second injection group confirms the fact that a change of the signal length does not lead to disturbances during signal generation or cause changes in the values of KWW or KWZ.

\subsection{Control of the signals assigned for the simultaneous injection}

Due to the fact that the frequency of generating the control signals (Fig. 3 and Fig. 4) for the simultaneous injection is the same with that in the case of the batched injection, it was necessary to introduce a change to the program (for the simultaneous injection with respect to the batched injection); a change of the port settings in the interrupts of the output ports. The modification of the hardware interface is not necessary because the signals can still be sent to set up the two groups of two injectors each. The control of the signals leading to the ignition coils remained the same as in the case of controlling the batched injection. Shown in Fig. 6 are two control signals that implement the program for the simultaneous injection for four cylinders. The presence of only two control signals is because the group of injectors has not been disconnected; only the signals controlling the two groups were triggered at the same time.

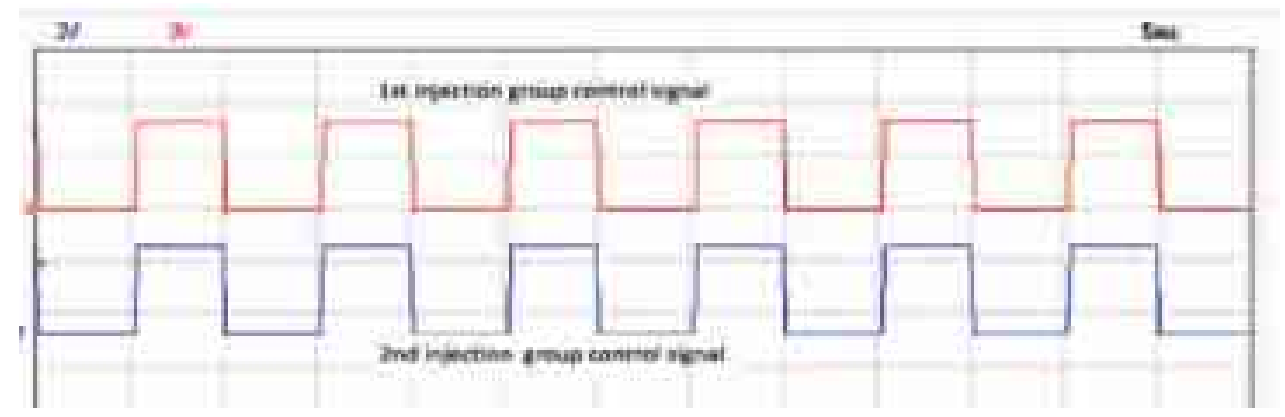

Fig. 6. An oscilloscope graph presenting the control signals for four cylinders for triggered simultaneously in two groups of two-injectors each

\subsection{Control signals for sequential injection}

For sequential injection, modifications in the hardware interface, as well as in the program are necessary. The frequency of the signals leading to the injectors is doubled, and the two groups of injectors, which were used for the simultaneous and batched injection, must be separated in order to generate signals to obtain injections before the opening of the intake valves of each cylinder separately.

On Fig. 7 are shown the interrupt signals of the crankshaft position sensor and control signals of the injectors of each particular cylinder during the sequential injection mode.

\section{Summary}

The controller presented in this paper can be used for research purposes during experiments performed on an engine test bench. It makes it possible to carry out series of tests, thereby it allows to determine how a modification of the injection and ignition parameters in combustion spark ignition engine with sequential, batched or simultaneous fuel injection affect the operating parameters and emission of toxic compounds etc. 


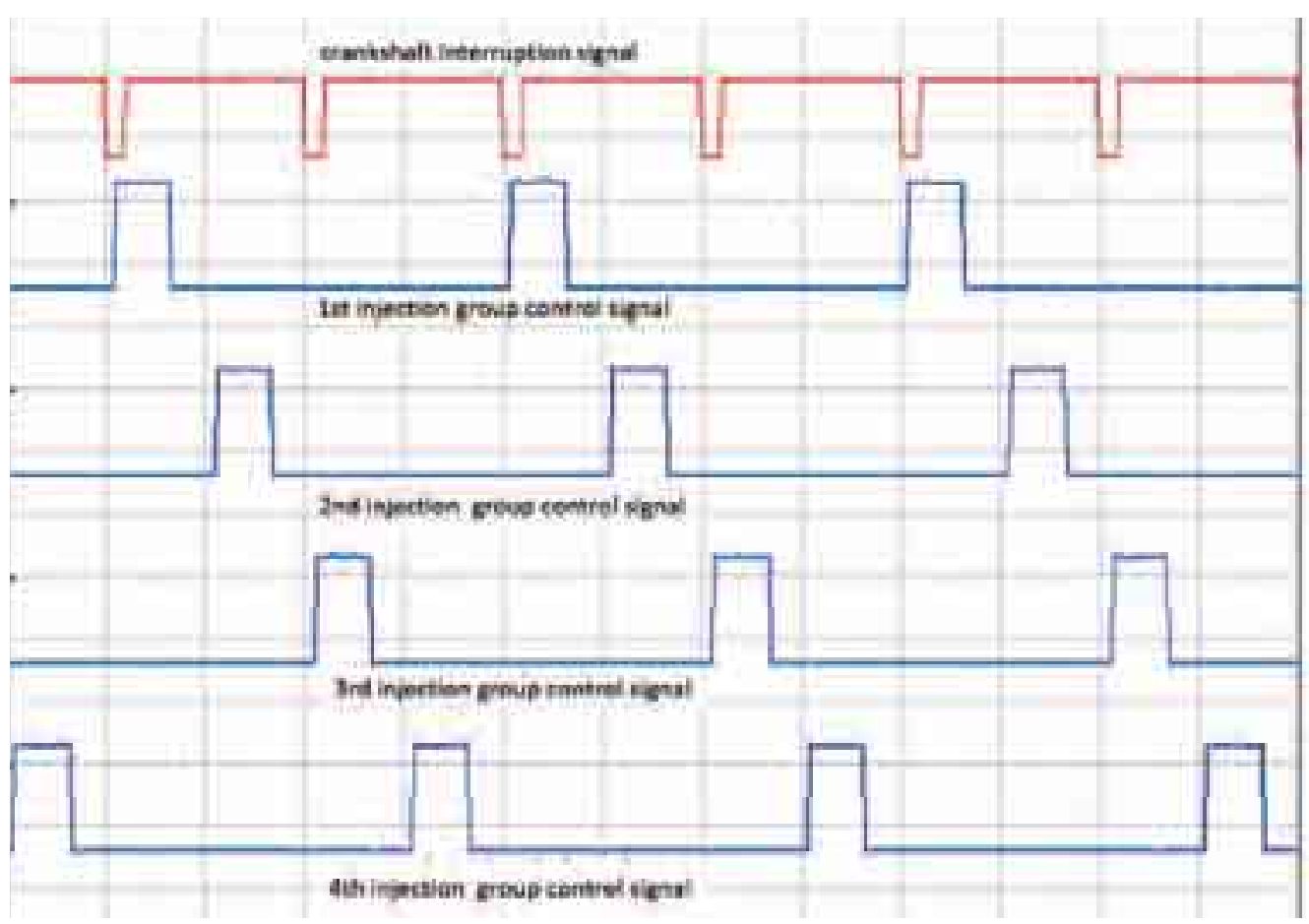

Fig. 7. An oscilloscope graph presenting control signals in sequential injection

The research carried out with this controller can help to verify computer simulations and calculations of the impact of control parameters in a combustion engine on particular output parameters.

The simulations shown in this article, concerning setting the values of parameters: KWW, KW, $\mathrm{KZ}$ and KWZ, through the interface, allows us to conclude that the signals controlling the injection and ignition angles of advance, as well as the signals controlling the length of the parameters of injection and ignition, are generated correctly.

The manner of adaptations to different modes of indirect injection, as shown in this article, proves that the controller has a wide-range of application during engine tests.

It is possible, through changes in the program and hardware interface, to adapt the controller to operate on a direct injection spark-ignition engine.

\section{References}

[1] Baranowski, R., Mikrokontrolery AVR ATmega w praktyce, Wydawnictwo BTC, Warszawa 2005.

[2] Doliński, J., Mikrokontrolery AVR w praktyce, Wydawnictwo BTC, Legionowo 2008.

[3] Gibek, Z., Bascom Basic AVR Help, MCS, 2005.

[4] Grochowska, K., Opracowanie sterownika do układu wtryskowego i zapłonowego silnika spalinowego, Master thesis, Rzeszów 2009.

[5] Kneba, Z., Makowski, S., Zasilanie i sterowanie silników, WKiŁ, Warszawa 2004.

[6] Wiązania, M., Programowanie mikrokontrolerów w języku Bascom, Wydawnictwo BTC, Warszawa 2004. 